\title{
COMPARISON OF A DOZEN AHP TECHNIQUES FOR GLOBAL VECTORS IN MULTIPERSON DECISION MAKING AND COMPLEX HIERARCHY
}

\author{
Stan Lipovetsky \\ GfK Custom Research North America \\ Minneapolis, MN, USA \\ E-mail: $\underline{\text { stan.lipovetsky@,gfk.com }}$
}

\begin{abstract}
A problem of synthesizing the local preferences into global priorities in multi-person decision making by the hierarchy structure is considered. Classic methods are tried together with new developed techniques, including multi-response multiplicative mode, three-dimensional eigenproblem and its simplification in parallel proportional profile estimation, orthogonal regression, nonlinear local evaluation, linear and nonlinear synthetic hierarchy optimizing procedures. Various marketing research data with different numbers of criteria, sub-criteria, alternatives, and respondents have been used to produce results by a dozen techniques. Most of the estimations yield rather similar global priority vectors with comparable ranks of the items. The best methods correspond to nonlinear estimations. The analysis performed helps in practical managerial decisions.
\end{abstract}

Keywords: AHP, multi-person decision making, complex hierarchy, global priority synthesis, optimizing.

\section{Introduction}

Analytic Hierarchy Process (AHP) is a methodology and a group of methods for multiple criteria decision making suggested and developed by Thomas Saaty (Saaty, 1977, 1980, 1994, 1996, 2000). AHP has been continuing to grow through the efforts of hundreds of authors in thousands of papers, and nowadays tons of theoretical and applied works on AHP can be found on the Internet, in books, and in professional journals in practically any area of human interests and activities (for instance, Golden et al., 1989; Wasil and Golden, 2003). Generally speaking, AHP is related to the family of Thurstone, Bradley-Terry, and other models of priority estimation via paired comparisons (MakKay et al., 1996; Lipovetsky and Conklin, 2001). But AHP has its own specifics in eliciting data from one or many experts by pair-wise ratio scales, arranging a hierarchical structure of comparisons by criteria, sub-criteria, and among alternatives, finding vectors of priorities within each group of the compared items, and composing local preferences by subcriteria and criteria of all hierarchy levels to get the synthesized global priorities among all the alternatives (Aczel and Saaty, 1983; Saaty and Kearns, 1985; Saaty and Vargas, 1984, 1994). Many specific modifications of AHP suggest optimizing procedures (Lipovetsky, 1996, 2005; Gass and Rapcsak, 2004), group evaluations by expert boards or multiple respondents (Basak and Saaty, 1993; Barzilai and Lootsma, 1997; Lipovetsky et al., 1997), statistical properties (Lipovetsky and Tishler, 1999), and robust and fuzzy priority estimations (Lipovetsky and Conklin, 2002; Enea and Piazza, 2004).

The current work studies various techniques of synthesizing global priorities in multi-person evaluations. A dozen classic and modern AHP tools have been implemented and compared. Besides the more known in group decision making multiplicative approach, I considered eigenvector analysis for threedimensional matrices of pairwise ratios by many experts. These kinds of eigenproblems in factor analysis had been studied in (Tucker, 1966; Law et al., 1984). This approach corresponds to the extension of the least squares AHP technique to the case of many experts, can be reduced to a non-linear eigenproblem, and simplified to the so called Parallel Proportional Profiles technique (Cattell, 1944; Lipovetsky, 1994; 
Lipovetsky and Tishler, 1994). Another approach utilizes a so called orthogonal regression technique constructed by the minimum distance of the observations to the theoretical surface (Maronna, 2005). It is also possible to approximate the original ratio relations in nonlinear priority modeling (Lipovetsky and Tishler, 1999). All these approaches at first produce the local preferences in group decisions, then convert them into the global priorities. Depending on the type of normalization (conventional or ideal) used for the local preference vectors, the global priority vectors can differ even for the same estimation technique. Besides these methods, simultaneous estimation of the global priority by all the data on the alternative, sub-criteria and criteria paired comparisons is employed via the so called synthetic hierarchy method (Lipovetsky, 1996, 2009). The numerical comparisons demonstrate that most of the methods produce similar vectors of preferences and their ranks, but the nonlinear estimations always have higher characteristics of the quality of fit, and can better serve the practical aims of managerial decision makers.

\section{Local and global priority vectors by one expert data}

Consider several methods and their sub-modes for the synthesized global priority evaluation.

\subsection{Classical AHP eigenvector}

At first let us briefly describe the main AHP relations. Each $i j$-th element in an AHP matrix is defined as a ratio of theoretical priorities $w_{i}$ and $w_{j}$ of the $i$-th and $j$-th items:

$$
W=\left(\begin{array}{cccc}
w_{1} / w_{1} & w_{1} / w_{2} & \ldots & w_{1} / w_{n} \\
--- & --- & --- \\
w_{n} / w_{1} & w_{n} / w_{2} & \ldots & w_{n} / w_{n}
\end{array}\right)
$$

Multiplying matrix (1) by the vector $w$ of priorities $w=\left(w_{1}, w_{2}, \ldots, w_{n}\right)^{\prime}$, yields the equalities:

$$
W w=n w .
$$

An elicited empirical pairwise ratios AHP matrix is

$$
A=\left(\begin{array}{cccc}
1 & a_{12} & \ldots & a_{1 n} \\
a_{21} & 1 & \ldots & a_{2 n} \\
\ldots \ldots & \ldots \ldots & \ldots \ldots & \ldots \ldots . . \\
a_{n 1} & a_{n 2} & \ldots & 1
\end{array}\right),
$$

with the transposed-reciprocal elements $a_{i j}=a_{j i}^{-1}$. By the pattern (2) the AHP relations for priority estimation are presented as an eigenproblem for the matrix (3):

$$
A \alpha=\lambda \alpha .
$$

The eigenvector $\alpha$ for the maximum eigenvalue $\lambda$ serves as the AHP estimate of the priorities. In some applications a vector of the transposed matrix is considered in the problem $A^{\prime} \beta=\lambda \beta$, that is interpreted as a vector of anti-priorities. An AHP priority vector is usually normalized by the total of its elements equals one - this is the conventional mode; or the vector is divided by its largest element - that is the so called ideal mode:

$$
\sum_{i} \alpha_{i}=1, \quad \alpha_{\max }=1
$$




\subsection{Multiplicative estimation}

Various other estimations of priority vectors are also known in the AHP. For instance, the multiplicative estimation is based on geometric means of the elements in each row of a pairwise ratio matrix. From the theoretical structure (1) we see that a product of the elements in an $i$-th row is proportional to $w_{i}^{n}$. So priority estimation by an empirical matrix (3) can be:

$$
\alpha_{i}=\left(\prod_{j=1}^{n} a_{i j}\right)^{1 / n},
$$

with additional normalizing by one of the modes (5).

\subsection{Least Squares}

The Least Squares approach corresponds to the singular value decomposition technique, and yields the eigenproblems:

$$
A A^{\prime} \alpha=\lambda \alpha, \quad A^{\prime} A \beta=\lambda \beta,
$$

where the main vector $\alpha$ estimates priorities, and the dual vector $\beta$ estimates anti-priorities.

\subsection{Synthesizing estimation}

In a more general case the AHP considers a hierarchy structure of criteria - subcriteria - alternatives, or even with more intermediate levels. The main goal consists in comparison of all the alternatives. The AHP estimates global priorities of the alternatives by synthesizing their local priorities with preference weights of the subcriteria and criteria. Let us denote a matrix $P$ of the primary criteria level $(j=1, \ldots, q-$ number of criteria), the matrices $S(k)$ of the secondary level of subcriteria (where $k=1, \ldots, m_{j}-$ the number of subcriteria within each criterion), and the matrices $T\left(n_{k}\right)$ of the tertiary level of alternatives $(t=$ $1, \ldots, n_{k}$ - the number of alternatives within each $k$-th subcriterion). AHP solves an eigenproblem of the kind (4) for each of such matrices:

$$
P \gamma=v \gamma, \quad S(k) \beta(k)=\mu(k) \beta(k), \quad T(t) \alpha(t)=\lambda(t) \alpha(t) .
$$

In (8) the eigenvalue and eigenvector of the primary criteria level are denoted as $v$ and $\gamma$, of the secondary level - as $\mu(k)$ and $\beta(k)$ for each $k$-th subcriterion, and for tertiary level - as $\lambda(t)$ and $\alpha(t)$ for a set of alternatives within each subcriterion, respectively. In each of the problems (8) we find the main eigenvector related to the largest eigenvalue, and use it as the estimate of the local priorities. Then we synthesize them into the global priorities taking each vector of local preferences, multiplying it by the corresponding element in the vector of the parent subcriterion and by the corresponding element in the vector of the parent criterion. Stacking all the weighted vectors of alternatives into one vector and normalizing it produces a global priority vector $\pi_{1}, \pi_{2} \ldots, \pi_{n}$ :

$$
\left(\begin{array}{l}
\pi_{1} \\
\pi_{2} \\
\ldots \\
\pi_{n}
\end{array}\right)=\left(\begin{array}{ccc}
\alpha_{1}^{1,1} & \ldots & \alpha_{1}^{m, 1} \\
\alpha_{2}^{1,1} & \ldots & \alpha_{2}^{m, 1} \\
\ldots & \ldots & \ldots \\
\alpha_{n}^{1,1} & \ldots & \alpha_{n}^{m, 1}
\end{array}\right)\left(\begin{array}{l}
\beta_{1}^{1} \\
\beta_{2}^{1} \\
\ldots \\
\beta_{m}^{1}
\end{array}\right) \gamma_{1}+\ldots+\left(\begin{array}{ccc}
\alpha_{1}^{1, q} & \ldots & \alpha_{1}^{m, q} \\
\alpha_{2}^{1, q} & \ldots & \alpha_{2}^{m, q} \\
\ldots & \ldots & \ldots \\
\alpha_{n}^{1, q} & \ldots & \alpha_{n}^{m, q}
\end{array}\right)\left(\begin{array}{l}
\beta_{1}^{q} \\
\beta_{2}^{q} \\
\ldots \\
\beta_{m}^{q}
\end{array}\right) \gamma_{q},
$$


where a system of $n$ alternatives are evaluated by each of $m$ subcriteria, and $q$ criteria. In (9), the columns $\alpha^{k, t}$ correspond to the alternatives' priority vectors with elements $\alpha_{t}^{k, j}$ of $t$-th alternatives related to the $k$ th parent subcriterion and $j$-th criterion, $\beta^{j}$ are the subcriteria's priority vectors within each $j$-th criterion, and the elements $\gamma_{1}, \ldots, \gamma_{q}$ are the criteria priorities. In a more complex structure of hierarchy, not all the alternatives but their different subsets can be estimated within each subcriterion, the same well as specific subsets of the subcriteria can be estimated within each criterion. Then column-vectors in the expression (9) should be arranged with zeros in place of the elements corresponding to the absent alternatives within subcriteria, or the absent subcriteria within the criteria.

The conventional or ideal mode (5) is used for all the vectors in the synthesizing process, and the resulting global priorities are related to the corresponding mode. In contrast to local preferences, the ranks of the global priorities can depend on the mode applied in the normalization of the local vectors.

\section{Local and global priority vectors in multi-person data}

Consider synthesizing global priorities by a hierarchy structure evaluated by many experts. At first let us describe the priorities obtained for each core of a hierarchy, when vectors of alternatives, subcriteria and criteria are normalized by one of the modes (5) and merged into global priorities by synthesizing (9).

\subsection{Multiplicative approach}

This technique is a straightforward extension of the geometric means (6) to incorporate data by many experts $(r=1, \ldots, N)$, so the priorities can be estimated as:

$$
\alpha_{i}=\left(\prod_{r=1}^{N} \prod_{j=1}^{n} a_{i j r}\right)^{\frac{1}{n N}},
$$

where each $a_{i j r}$ is an $i j$-th element of a matrix (3) related to the $r$-th respondent. Instead of the estimation (10), for each AHP matrix we can construct a regression model of the logarithm of the ratios by the set of dummy variables indicating by +1 and -1 the item preferred to its counterpart in their pair comparison (the technique described for a similar estimation of Thurstone scale in Lipovetsky and Conklin, 2004). Fixing one of the items (taking its regression coefficient equals zero) we obtain a multiple linear regression by the other items, then we stack the regression coefficients with the fixed zero coefficient, use exponentiation of them, and normalize by one of the conditions (5). It produces the same results as the formula (10) yields, but the regression approach also gives $t$-statistics to estimate the difference of the preferences from the fixed one, coefficient of multiple determination, its $p$-value, and other characteristics of the quality of fit.

\subsection{Three-Dimensional Eigenproblem}

Consider a three-dimensional matrix arranged by piling the pairwise ratios matrices (3) of many experts. Then in place of the regular eigenproblem (4) we have an eigenproblem for a 3D-matrix where the layers present data elicited from different respondents. The solution can be obtained in the Least Squares (LS) approximation of the 3D-matrix with elements $a_{i j r}$ (as in (10)) by the outer product of the vectors $a, b$, and $c$ corresponding to each of three directions of the matrix:

$$
L S=\sum_{i j r}\left(a_{i j r}-\lambda a_{i} b_{j} c_{r}\right)^{2} .
$$

Differentiating this objective by the vectors' elements yields a system of equations: 


$$
\sum_{j r} a_{i j r} b_{j} c_{r}=\lambda a_{i}, \quad \sum_{i r} a_{i j r} a_{i} c_{r}=\lambda b_{j}, \quad \sum_{i j} a_{i j r} a_{i} b_{j}=\lambda c_{r},
$$

which is a three-dimensional extension of the relations known in the singular value decomposition technique. Substitution of the vector $c$ from the last relation (12) into the two others yields a non-linear eigenproblem:

$$
\left(\begin{array}{cc}
Q(b) Q^{\prime}(b) & 0 \\
0 & Q(a) Q^{\prime}(a)
\end{array}\right)\left(\begin{array}{l}
a \\
b
\end{array}\right)=\lambda^{2}\left(\begin{array}{l}
a \\
b
\end{array}\right),
$$

where the following notations for the matrices are used:

$$
(Q(b))_{i r}=\sum_{j} a_{i j r} b_{j}, \quad(Q(a))_{j r}=\sum_{i} a_{i j r} a_{i} .
$$

Solution (13) consists of the vector $a$ of priorities and a dual vector $b$ of anti-priorities, and the problem (13)(14) is a generalization of the method (7) to the multiple experts. Characteristics of the fitting quality in this approach (11)-(14) are considered in (Lipovetsky, 1994; Lipovetsky and Tishler, 1994).

\subsection{PPP-solution for 3D-eigenvector}

There is a simpler way to estimate the main eigenvectors for a 3D-matrix. The results (13)-(14) can be simplified due to the Parallel Proportional Profiles principle applied to a 3D-matrix. Then the elements $a_{i}$ of the vector $a$ for the 3-dimensional matrix are approximately proportional to the largest singular values of the 2-dimensional matrices identified by the $i$-th sections. The same is true for other vectors: the components $b_{j}$ of the vector $b$ in (13) are approximately proportional to the largest singular values of 2dimensional matrices identified by the fixed $j$-th layer of the 3D-matrix. Similarly we can estimate the components $c_{r}$ of the vector $c$ in (11)-(12) by the largest singular values of 2-dimensional matrices of the $r$-th respondent. It means that for estimation of the first element of a priority vector $a$, we can take the first rows of the matrices (3) for each respondent, stack them into a matrix of $n$ by $N$ order, and find its largest singular value $v_{1}^{\max }$. Then repeat this procedure with the second rows of the matrices (3) by all respondents, finding the largest singular value $v_{2}^{\max }$, etc. Combining all these maximum values into one vector, we obtain an estimate for the priority vector of the 3-dimensional matrix:

$$
a=\left(v_{1}^{\max }, v_{2}^{\max }, \ldots, v_{n}^{\max }\right) \text {. }
$$

So the estimate of the main eigenvector of a 3-dimensional matrix is reduced to a set of the eigenvalues of the regular 2-dimensional matrices.

\subsection{Orthogonal Regression}

Each element in a matrix (3) expresses the ratio of two priorities (1), that for many respondents can be presented in a linearized form as

$$
\alpha_{i}=a_{i j r} \alpha_{j}+\varepsilon_{i j r},
$$

where the elements $a_{i j r}$ include the index for each $r$-th respondent, $\alpha_{i}$ are the estimates of the theoretical preferences, and $\varepsilon_{i j r}$ are the error terms in this model. Expressing one item via all the others, we can apply regression modeling for estimation of the priorities. However, these priorities depend on the choice of the item regressed by all the rest of them, and if there are $n$ items $n$ different models can be constructed. It is better to employ an orthogonal regression constructed by the minimum distance of the observations to the theoretical surface. It produces a unique relation of all the variables in one model. Solution can be found as the eigenvector corresponded to the minimum eigenvalue (minimum sum of squared distances from observations to the theoretical surface by perpendicular).

\subsection{Nonlinear Regression}

In place of the linearized model (16) we can use the original ratio relation of any two priorities (1), so for many respondents we have a model: 


$$
a_{i j r}=\alpha_{i} / \alpha_{j}+\varepsilon_{i j r}
$$

Minimizing the error terms $\varepsilon_{i j r}$ corresponds to the nonlinear regression model of the elicited ratio values on the left-hand side by all the priority estimates $\alpha_{i}$ at the right-hand side (17), in the assumption of the same theoretical preferences across the respondents. This problem is easy to solve by nonlinear estimations using any modern statistical software package. In contrast to the multiplicative approach (10) corresponding to the assumption of the relative error term multiplied by the elicited priority ratio, the model (17) matches to the assumption of the additive errors for the elicited paired ratios (Lipovetsky and Tishler, 1999).

All the techniques described above in this section, produce group priorities for each core of the hierarchical structure, then the vectors are normalized by one or the other conditions (5) and synthesized by (9) into the global priorities. The results would depend on the mode of the vectors normalization, so for each of the five described techniques we actually obtain two solutions of conventional and ideal modes (5).

\subsection{Synthetic hierarchy evaluation}

Consider approaches based on simultaneous operating with all the data from the alternatives, sub-criteria and criteria paired comparisons. Such techniques from the beginning employ the global priorities, and produce results independent of the normalizing modes (Lipovetsky, 1996). In place of the local priority vectors it is possible to use elements of a global vector constructed by the items corresponding to all the alternatives, subcriteria and criteria. The elements $\pi_{1}, \pi_{2} \ldots, \pi_{n}$ of the global priority vector (9) can be represented as totals in each row of the following matrix:

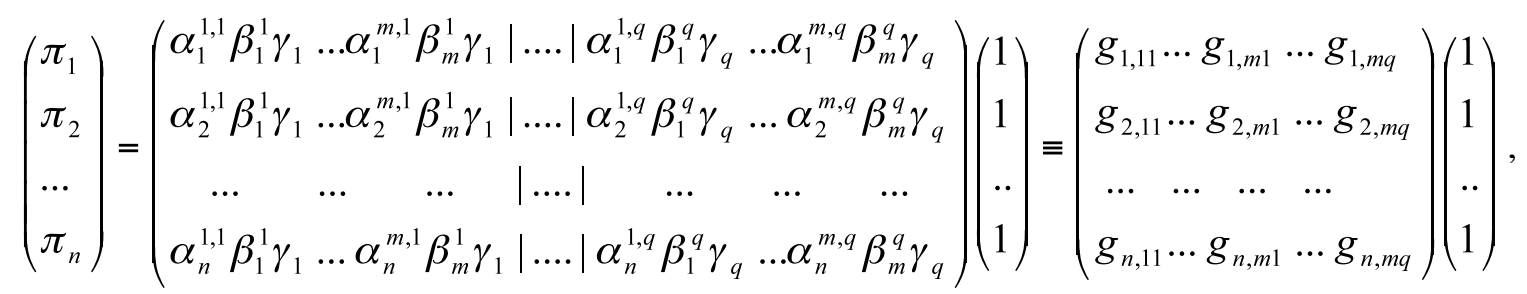

where we incorporate the elements of the vectors $\beta^{j}$ of sub-criteria and $\gamma_{1}, \ldots, \gamma_{q}$ of criteria into the local alternative preferences $\alpha_{t}^{k, j}$ of each $t$-th alternative related to the $k$-th parent sub-criterion and $j$-th criterion. Such weighted items of local alternatives' preferences denoted by $g_{t, k j}$ arrange a matrix (18) of $n$ by $M$ order, where $n$ is the number of alternatives and $M=\sum_{k}^{q} m_{k}$ is a total number of all sub-criteria by all criteria (8). Sub-matrices of the sub-criteria related to the same criterion are marked by the vertical lines in the matrix of the weighted preferences in (18).

It is clear from the structure of the elements in the matrices (18) that a paired ratio of the alternatives within any sub-criterion and criterion (that is a column in these matrices) can be expressed either by the quotient of the local priorities (as in (1) or (17)), or by the quotient of the weighted priorities. Thus, for any $k$-th parent sub-criterion and $j$-th criterion, a proportion of preferences between $t$-th and $l$-th alternatives is held:

$$
\frac{g_{t, k j}}{g_{l, k j}}=\frac{\alpha_{t}^{k, j} \beta_{k}^{j} \gamma_{j}}{\alpha_{l}^{k, j} \beta_{k}^{j} \gamma_{j}}=\frac{\alpha_{t}^{k, j}}{\alpha_{l}^{k, j}} .
$$

Similarly, the quotient of totals across the elements in two columns of the matrices (18) corresponding to any two $k$-th and $p$-th sub-criteria of the same $j$-th criterion equals: 


$$
\frac{\sum_{t=1}^{n} g_{t, k j}}{\sum_{l=1}^{n} g_{l, p j}}=\frac{\sum_{t=1}^{n} \alpha_{t}^{k j} \beta_{k}^{j} \gamma_{j}}{\sum_{l=1}^{n} \alpha_{l}^{p j} \beta_{p}^{j} \gamma_{j}}=\frac{\beta_{k}^{j} \gamma_{j} \sum_{t=1}^{n} \alpha_{t}^{k j}}{\beta_{p}^{j} \gamma_{j} \sum_{l=1}^{n} \alpha_{l}^{p j}}=\frac{\beta_{k}^{j}}{\beta_{p}^{j}}
$$

where the conventional mode (5) is used for the local alternatives' preferences $\alpha_{t}^{k, j}$. Finally, the quotient of totals by all the elements in two sub-matrices of the matrix (18) corresponding to any two $j$-th and $s$-th criteria is:

$$
\frac{\sum_{k=1}^{m} \sum_{t=1}^{n} g_{t, k j}}{\sum_{p=1}^{m} \sum_{l=1}^{n} g_{l, p s}}=\frac{\gamma_{j} \sum_{k=1}^{m} \beta_{k}^{j} \sum_{t=1}^{n} \alpha_{t}^{k j}}{\gamma_{s} \sum_{p=1}^{m} \beta_{p}^{s} \sum_{l=1}^{n} \alpha_{l}^{k j}}=\frac{\gamma_{j} \sum_{k=1}^{m} \beta_{k}^{j}}{\gamma_{s} \sum_{p=1}^{m} \beta_{p}^{s}}=\frac{\gamma_{j}}{\gamma_{s}},
$$

where the conventional mode (5) is applied for normalizing the local alternatives' preferences $\alpha_{t}^{k, j}$ and the sub-criteria preferences $\beta_{k}^{j}$.

These relations show that the paired ratios of the alternatives, sub-criteria, and criteria can be approximated by ratios of the synthesized priorities $g_{t, k j}$ (19), of their totals within each sub-criterion (20), and of their totals within each criterion (21). So the parameters of the synthesized priorities can be consistently used for fitting the elicited paired comparison data of all the levels and centers of the hierarchy structure. Let us describe another exposition of such techniques in a form more convenient for practical applications.

\subsection{Designing the whole data matrix}

Similarly to the regression approach discussed after the formula (10), we arrange a design matrix of $n$ independent dummy variables - each of them identifies one of total $n$ alternatives. Taking a matrix of paired ratios for a subset of alternatives related to one of the sub-criteria, we transform its data into the elements of the design matrix. Each row the design matrix would contain one element +1 and another -1 indicating the pair compared items and the item preferred to its counterpart, respectively. All other entries in this row equal zero. The related vector-column of the response would have in this row the value of the paired ratio itself. After stacking all the rows of the paired comparison among the alternatives, we add the rows of the paired comparisons among the sub-criteria. In accordance with the sums at the left-hand side (20), in each row of the design matrix we take so many elements +1 and so many elements -1 as many alternatives $n_{k}$ and $n_{p}$ belong to the superior and inferior sub-criteria, respectively. Actually, to balance the influence of each subcriterion, we use not ones, but those divided by these $n_{k}$ and $n_{p}$ numbers. After that we add the rows corresponding to the criteria comparisons. Due to the sums at the left-hand side (21), in the rows of the design matrix we take the elements +1 and -1 in all the places of the alternatives entering into the sub-criteria that belong to the superior and inferior criteria, respectively. Again, to balance the influence of these elements, we use not ones or minus ones, but their mean values.

For illustration, consider an AHP structure with two criteria $A$ and $C$, two sub-criteria $a$ and $b$ within the criterion $A$, three alternatives $\left(a_{1}, a_{2}, a_{3}\right)$ within the sub-criterion $a$, two alternatives $\left(b_{1}, b_{2}\right)$ within the subcriterion $b$, and three alternatives $\left(c_{1}, c_{2}, c_{3}\right)$ within the criterion $C$, respectively. Suppose elicited from an expert the AHP matrices of paired ratios within each group of the alternatives are:

$$
\left(\begin{array}{ccc}
1 & a_{12} & a_{13} \\
a_{21} & 1 & a_{23} \\
a_{31} & a_{32} & 1
\end{array}\right)=\left(\begin{array}{ccc}
1 & 9 & 5 \\
1 / 9 & 1 & 1 / 3 \\
1 / 5 & 3 & 1
\end{array}\right), \quad\left(\begin{array}{ll}
1 & b_{12} \\
b_{21} & 1
\end{array}\right)=\left(\begin{array}{cc}
1 & 7 \\
1 / 7 & 1
\end{array}\right), \quad\left(\begin{array}{lll}
1 & c_{12} & c_{13} \\
c_{21} & 1 & c_{23} \\
c_{31} & c_{32} & 1
\end{array}\right)=\left(\begin{array}{ccc}
1 & 6 & 2 \\
1 / 6 & 1 & 1 / 2 \\
1 / 2 & 2 & 1
\end{array}\right),
$$

and the matrices of pair comparisons between the sub-criteria $a$ and $b$, and also between criteria $A$ and $C$ are as follows: 


$$
\left(\begin{array}{cc}
1 & a_{a b} \\
a_{b a} & 1
\end{array}\right)=\left(\begin{array}{cc}
1 & 8 \\
1 / 8 & 1
\end{array}\right), \quad\left(\begin{array}{cc}
1 & a_{A C} \\
a_{C A} & 1
\end{array}\right)=\left(\begin{array}{cc}
1 & 1 / 4 \\
4 & 1
\end{array}\right) .
$$

The design matrix of all $n=8$ alternatives is presented in Table 1 by dummy variables $x_{1, \ldots}, x_{8}$, and the response $y$. In its first three numerical rows the data from the first matrix (22a) is presented. For instance, the $a_{12}=9$ element shown by +1 and -1 in places where the compared items $a_{1}$ and $a_{2}$, all the other elements are zeros, and the ratio 9 is given in the last column of the response. We don't need to present the complementary value of $a_{21}$ in the design matrix. The fourth row shows the ratio $b_{12}=7$ from the second matrix, and the next three rows give the data from the third matrix in (22a). After that we add to Table 1 a row with data of the $a$ and $b$ sub-criteria comparison (the first matrix (22b)) - there are $+1 / 3$ values for three elements belonging to the first sub-criterion, and $-1 / 2$ for the elements related to the second one. The last row of Table 1 consists of the values $-1 / 5$ for five elements entering under the first criterion, and of $+1 / 3$ for three elements entering under the second criterion.

Table 1. Hierarchy Design Matrix of Pair Comparisons by an Expert.

\begin{tabular}{|c|c|c|c|c|c|c|c|c|c|}
\hline \multirow{4}{*}{ Hierarchy } & \multicolumn{5}{|c|}{ Criterion $A$} & \multicolumn{3}{|c|}{ Criterion $C$} & \multirow{2}{*}{$\begin{array}{c}\text { Pair } \\
\text { Comparison }\end{array}$} \\
\hline & \multicolumn{3}{|c|}{ Sub-criterion $a$} & \multicolumn{2}{|c|}{ Sub-criterion $b$} & & - & & \\
\hline & \multicolumn{3}{|c|}{ Alternatives } & \multicolumn{2}{|c|}{ Alternatives } & \multicolumn{3}{|c|}{ Alternatives } & Ratio \\
\hline & $a_{1}$ & $a_{2}$ & $a_{3}$ & $b_{1}$ & $b_{2}$ & $c_{1}$ & $c_{2}$ & $c_{3}$ & Response \\
\hline Pairldummy & $x_{1}$ & $x_{2}$ & $x_{3}$ & $x_{4}$ & $x_{5}$ & $x_{6}$ & $x_{7}$ & $x_{8}$ & $y$ \\
\hline$a_{12}$ & 1 & -1 & 0 & 0 & 0 & 0 & 0 & 0 & 9 \\
\hline$a_{13}$ & 1 & 0 & -1 & 0 & 0 & 0 & 0 & 0 & 5 \\
\hline$a_{23}$ & 0 & 1 & -1 & 0 & 0 & 0 & 0 & 0 & $1 / 3$ \\
\hline$b_{12}$ & 0 & 0 & 0 & 1 & -1 & 0 & 0 & 0 & 7 \\
\hline$c_{12}$ & 0 & 0 & 0 & 0 & 0 & 1 & -1 & 0 & 6 \\
\hline$c_{13}$ & 0 & 0 & 0 & 0 & 0 & 1 & 0 & -1 & 2 \\
\hline$c_{23}$ & 0 & 0 & 0 & 0 & 0 & 0 & 1 & -1 & 0.5 \\
\hline$a b$ & $1 / 3$ & $1 / 3$ & $1 / 3$ & $-1 / 2$ & $-1 / 2$ & 0 & 0 & 0 & 8 \\
\hline$A C$ & $-1 / 5$ & $-1 / 5$ & $-1 / 5$ & $-1 / 5$ & $-1 / 5$ & $1 / 3$ & $1 / 3$ & $1 / 3$ & 4 \\
\hline
\end{tabular}

Note that we can change signs of all the elements in any row, but simultaneously invert the ratio value at the response column. Having the data of many experts, we stack their Table 1 matrices in rows into one extended matrix of all respondents. In a matrix of Table 1 the total in each row equals zero, so this matrix has rank $n-1$, and one column can be excluded for regression fitting of the response $y$ by the set of dummy variables (a similar technique for Thurstone scaling is described in Lipovetsky and Conklin, 2004).

\subsection{Linear regression by all the data}

Suppose a total matrix similar to Table 1 by all the paired comparison AHP matrices from all the hierarchy centers and by all respondents is constructed. Such data is ready for regression modeling of the logarithm of the ratio scores in the last column by the dummy variables (taking $n-1$ of them, except any redundant one), and without intercept:

$$
\ln y_{i}=d_{1} x_{i 1}+d_{1} x_{i 2}+\ldots+d_{1} x_{i, n-1}+\delta_{i}
$$

where fitting is performed by the least squares minimizing of the error terms $\delta_{i}$, and index $i$ corresponds to the rows of observations in Table 1 . When the regression coefficients $d$ are estimated, we add a zerocoefficient in place of the excluded dummy variable, take the exponent of all coefficients, and normalize them, say, by the conventional mode (5). The obtained vector presents the global priorities in the simultaneous multiple linear regression estimation by all the hierarchy levels and all the respondents. This regression corresponds to minimizing the deviations in models similar to (15)-(16) but with the multiplicative relative error term. It also uses the multiplicative in place of the additive combinations of the weighted 
preference ratios on the left-hand side in (20)-(21), when taking the logarithm transforms the models to the linearized form (23). The results of estimation (23) do not depend on which dummy variable was excluded in the modeling. Besides the coefficients transformed to the priorities, the regression (23) yields their errors and $t$-statistics which help estimate the significance of any preference in comparison with the fixed one, and other characteristics of the model's quality.

\subsection{Nonlinear regression by all the data}

Nonlinear fitting of all the data can be performed by the following model:

$$
y_{i}=\left(\sum_{j=1}^{n} d_{j} x_{i j}\left[x_{i j}>0\right]\right) /\left(\sum_{j=1}^{n} d_{j} x_{i j}\left[x_{i j}<0\right]\right)+\varepsilon_{i},
$$

where each $x_{i j}$ corresponds to the element in $i$-th row and $j$-th column of a matrix in Table 1 . Notations $x_{i j}\left[x_{i j}>0\right]$ and $x_{i j}\left[x_{i j}<0\right]$ indicate that the positive and the negative inputs from the dummy variables $x_{i j}$ in Table 1 are taken in the numerator and denominator (24), respectively. Parameters $d_{j}$ are the estimators of the global priorities, and the model (24) corresponds to the relations (19)-(21) expressing the paired ratios via the weighted preferences. Because a matrix of Table 1 has rank $n-1$, and the nonlinear form (24) is homogeneous by the estimated parameters, one of them should be fixed, for instance we can assign $d_{1}=1$. The parameters can be found via the nonlinear minimization of the errors $\varepsilon_{i}$, which can be achieved using software available in modern statistical packages. The estimated parameters can be standardized to the priorities by the conventional mode (5).

\subsection{Quality of fit}

In any global priority vector, taking the elements corresponding to a particular subset of pair-compared alternatives and normalizing them by a mode (5), we obtain the vector of local priorities for this center of the hierarchy. Similarly, picking the subsets of the global priority elements corresponding to the subcriteria or criteria, finding their totals (due to (20) for sub-criteria, or (21) for criteria), and normalizing them by (5), we obtain the local vectors of priority within the sub-criteria or criteria, respectively. To check the quality of approximation of all the pair comparison matrices by the obtained global priority vectors it is convenient to use the coefficient of multiple determination defined similarly to the one well known in regression modeling:

$$
R^{2}=1-\frac{S_{\text {residual }}^{2}}{S_{\text {equivalent }}^{2}}=\frac{\sum_{\text {criteria }} \sum_{\text {subcriteria }} \sum_{\text {alternatives respondents }} \sum_{\text {criteria }} \sum_{\text {subcriteria }} \sum_{\text {alternatives respondents }} \sum_{j k}\left(a_{j}-1\right)^{2}}{\left.\sum_{j} \alpha_{k}\right)^{2}},
$$

where $a_{j k}$ corresponds to a pair-wise ratio of two items in any data matrix of alternatives, sub-criteria, and criteria comparisons, and $\alpha_{j} / \alpha_{k}$ is this ratio estimated by the found priority vectors with the elements $\alpha_{j}$, and the summing is performed by the paired comparisons in all levels of the hierarchy structure. In numerator (25) we have the total residual sum of squares $S_{\text {residual }}^{2}$ of the estimated priorities ratio deviations from the elicited values. In denominator (25) we have the total equivalent sum of squares $S_{\text {equivalent }}^{2}$ using the assumption of the same preferences in any ratio deviations from the elicited values. So the coefficient (25) shows how much the found priorities outperform a hypothesis of the same preferences by all alternatives. The better (worse) is the approximation - the smaller (larger) is the residual sum of squares, and the closer is the coefficient of multiple determination (25) to one (to zero). 


\section{Comparison of the numerical results}

Consider an example from a real project in marketing research with the alternatives corresponding to a variety of products. There are two primary criteria (denoted $A$ and $B$ ), six secondary sub-criteria within $A$ (those are $a, b, c, d, e$ and $f$ ) and three secondary sub-criteria within $B$ (those are $x, y$, and $z$ ). Within these sub-criteria there are the following numbers of tertiary items given in parentheses: $a(3), b(5), c(5), d(4)$, $e(3), f(3)$, also $x$ (4), $y(2)$, and $z$ (4). All 33 items can be denoted using criterion, sub-criterion and item notations as follows: $A a 1, A a 2, A a 3, A b 1, \ldots, A f 3$, and $B x 1, \ldots, B z 3$. Each of ten experts evaluated the following paired relations for this hierarchy: 1 pair $(A$ and $B)$ on the upper level; 15 pairs among $(a, b, c$, $d, e$ and $f$ ), etc, for a total of 67 comparisons elicited and arranged in the AHP matrices of paired ratios for 10 experts. The global priority vectors estimated by various techniques are presented in Table 2 .

Table 2: Scores of Global Priority in Various Estimations.

\begin{tabular}{|c|c|c|c|c|c|c|c|c|c|c|c|c|c|}
\hline \multicolumn{2}{|c|}{ Item } & \multicolumn{2}{|c|}{$\begin{array}{c}\text { Multiplicative } \\
\text { method }\end{array}$} & \multicolumn{2}{|c|}{$\begin{array}{c}\text { 3D- } \\
\text { eigenvector }\end{array}$} & \multicolumn{2}{|c|}{$\begin{array}{c}\text { PPP- } \\
\text { eigenvector }\end{array}$} & \multicolumn{2}{|c|}{$\begin{array}{c}\text { Orthogonal } \\
\text { regression }\end{array}$} & \multicolumn{2}{|c|}{$\begin{array}{l}\text { Nonlinear } \\
\text { regression }\end{array}$} & \multirow{2}{*}{$\begin{array}{c}\begin{array}{c}\mathrm{SH} \\
\text { linear }\end{array} \\
\text { conv }\end{array}$} & \multirow{2}{*}{$\begin{array}{c}\mathrm{SH} \\
\text { nonlin } \\
\text { conv }\end{array}$} \\
\hline$\#$ & name & conv & ideal & conv & ideal & conv & ideal & conv & ideal & conv & ideal & & \\
\hline 1 & $A a 1$ & 15.2 & 11.5 & 12.2 & 9.7 & 8 & 6.6 & 41.2 & 36.3 & 18 & 14.2 & 16 & 17 \\
\hline 2 & $A a 2$ & 2.9 & 2.2 & 3.4 & 2.7 & 3.6 & 3 & 4.8 & 4.2 & 3.1 & 2.5 & 3.1 & 4.3 \\
\hline 3 & $A a 3$ & 2.9 & 2.2 & 4.2 & 3.3 & 3.9 & 3.3 & 5 & 4.4 & 3 & 2.5 & 3 & 2.9 \\
\hline 4 & $A b 1$ & 3.3 & 6 & 2.6 & 3.9 & 2.4 & 3.6 & 3.9 & 4.8 & 1.2 & 1.3 & 4.5 & 1 \\
\hline 5 & $A b 2$ & 1.5 & 2.7 & 1.2 & 1.7 & 1.9 & 2.8 & 0.7 & 0.8 & 0.6 & 0.7 & 2.1 & 0.7 \\
\hline 6 & $A b 3$ & 2.4 & 4.4 & 2.6 & 3.8 & 2.6 & 3.8 & 1.1 & 1.4 & 0.3 & 0.3 & 3.3 & 0.2 \\
\hline 7 & $A b 4$ & 2.4 & 4.3 & 3.8 & 5.6 & 3.4 & 5 & 0.7 & 0.9 & 2.8 & 3.1 & 3.2 & 3 \\
\hline 8 & $A b 5$ & 1.2 & 2.1 & 1.2 & 1.8 & 1.4 & 2.1 & 0.3 & 0.4 & 0.4 & 0.4 & 1.6 & 0.4 \\
\hline 9 & $A c 1$ & 2.6 & 4.2 & 3.1 & 4.9 & 2.8 & 4.3 & 1.6 & 1.9 & 3.6 & 5.6 & 3.5 & 3.6 \\
\hline 10 & $A c 2$ & 1.8 & 2.9 & 1.5 & 2.3 & 2.3 & 3.6 & 0.6 & 0.7 & 1 & 1.6 & 2.4 & 1 \\
\hline 11 & Ac3 & 4.4 & 7.2 & 4.9 & 7.8 & 4.3 & 6.6 & 5.1 & 6.2 & 7.1 & 11 & 6 & 6.8 \\
\hline 12 & $A c 4$ & 2.7 & 4.4 & 4.1 & 6.4 & 3.6 & 5.6 & 0.9 & 1.1 & 5.3 & 8.3 & 3.6 & 5.1 \\
\hline 13 & Ac5 & 1.6 & 2.6 & 2.3 & 3.5 & 2.6 & 4 & 0.6 & 0.7 & 1.3 & 2 & 2.2 & 1.2 \\
\hline 14 & $A d 1$ & 6.9 & 8.1 & 8.7 & 9.9 & 6.3 & 7.4 & 4.5 & 4.8 & 12 & 14.5 & 7.8 & 12 \\
\hline 15 & $A d 2$ & 3 & 3.5 & 4.1 & 4.6 & 4 & 4.7 & 0.8 & 0.9 & 2.1 & 2.6 & 3.3 & 2 \\
\hline 16 & $A d 3$ & 2.1 & 2.4 & 2.6 & 3 & 2.6 & 3 & 0.7 & 0.8 & 2.2 & 2.7 & 2.3 & 2.3 \\
\hline 17 & $A d 4$ & 2.8 & 3.3 & 4.8 & 5.4 & 4.2 & 4.9 & 0.8 & 0.9 & 7.7 & 9.3 & 3.1 & 7.7 \\
\hline 18 & $A e 1$ & 6.7 & 4.9 & 7.3 & 5.4 & 6.1 & 4.6 & 4.3 & 3.8 & 4.7 & 3.7 & 7.5 & 4.9 \\
\hline 19 & $A e 2$ & 1.2 & 0.8 & 1.8 & 1.3 & 2.3 & 1.8 & 0.5 & 0.4 & 0.8 & 0.6 & 1.3 & 0.8 \\
\hline 20 & Ae3 & 1 & 0.7 & 1.9 & 1.4 & 2.3 & 1.8 & 0.5 & 0.4 & 0.7 & 0.5 & 1.2 & 0.7 \\
\hline 21 & Afl & 3.2 & 3.7 & 3.6 & 3.2 & 2.7 & 3 & 1.7 & 3.3 & 3.7 & 3 & 2.5 & 3.6 \\
\hline 22 & Af2 & 1.8 & 2 & 1 & 0.8 & 2.5 & 2.8 & 1.8 & 3.6 & 0.6 & 0.5 & 1.4 & 0.5 \\
\hline 23 & $A f 3$ & 1.9 & 2.2 & 1.9 & 1.6 & 1.8 & 2.1 & 1.6 & 3.3 & 0.8 & 0.7 & 1.5 & 0.8 \\
\hline 24 & $B x 1$ & 8.1 & 3.7 & 4 & 1.8 & 4.3 & 2.1 & 8.7 & 7.2 & 5.5 & 3 & 5.3 & 5.6 \\
\hline 25 & $B \times 2$ & 2.4 & 1.1 & 1.6 & 0.7 & 2.9 & 1.4 & 1.3 & 1.1 & 1.2 & 0.7 & 1.5 & 1.3 \\
\hline 26 & $B \times 3$ & 2.2 & 1 & 1.3 & 0.6 & 2.3 & 1.1 & 1.1 & 0.9 & 0.9 & 0.5 & 1.5 & 0.9 \\
\hline 27 & $B x 4$ & 2.6 & 1.2 & 2.1 & 1 & 2.9 & 1.4 & 1.1 & 0.9 & 3.9 & 2.1 & 1.7 & 3.8 \\
\hline 28 & By1 & 1.7 & 0.6 & 0.7 & 0.2 & 2.4 & 0.6 & 0.6 & 0.4 & 0.6 & 0.2 & 0.5 & 0.6 \\
\hline 29 & By2 & 3.7 & 1.3 & 3.8 & 0.9 & 4.8 & 1.2 & 2.1 & 1.6 & 2.6 & 0.9 & 1.1 & 2.7 \\
\hline 30 & $B z 1$ & 1.2 & 0.9 & 0.6 & 0.3 & 0.8 & 0.5 & 1 & 1 & 0.4 & 0.2 & 0.7 & 0.4 \\
\hline 31 & $B z 2$ & 1 & 0.7 & 0.5 & 0.3 & 0.8 & 0.5 & 0.4 & 0.4 & 1.8 & 0.7 & 0.6 & 2 \\
\hline 32 & $B z 3$ & 0.7 & 0.5 & 0.2 & 0.1 & 0.6 & 0.4 & 0.2 & 0.2 & 0.1 & 0 & 0.4 & 0.1 \\
\hline 33 & $B z 4$ & 0.7 & 0.5 & 0.4 & 0.2 & 0.6 & 0.4 & 0.2 & 0.2 & 0.3 & 0.1 & 0.4 & 0.3 \\
\hline & $\kappa$ & 0.23 & 0.23 & 0.24 & 0.24 & 0.13 & 0.14 & 0.04 & 0.13 & 0.38 & 0.39 & 0.25 & 0.38 \\
\hline
\end{tabular}


The first two vectors correspond to the multiplicative method (10), with both conventional and ideal modes (5) used within each local priority (the modes are denoted as conv and ideal in Table 2). For the sake of comparison, the global vectors obtained with the ideal mode are finally renormalized to the conventional mode. Also, the vectors are multiplied by 100 , so their elements are the priority percent shares. The next pairs of vectors in Table 2 are estimated by 3D- eigenvector problem (13), by the simplified PPP-solution (15), by the orthogonal regression (16), and by nonlinear regression (17). Finally, the synthetic hierarchy simultaneous estimations (18)-(21) of all global priorities in the linear (23) and nonlinear (24) approximations are presented in the last two columns of Table 2 . The coefficient $R^{2}(25)$ in the last row of Table 2 indicates that the best results are suggested by the nonlinear models. Next Table 3 presents the ranks of priorities from Table 2 .

Table 3: Ranks of Global Priority in Various Estimations.

\begin{tabular}{|c|c|c|c|c|c|c|c|c|c|c|c|c|c|}
\hline \multicolumn{2}{|c|}{ Item } & \multicolumn{2}{|c|}{$\begin{array}{l}\text { Multiplicative } \\
\text { method }\end{array}$} & \multicolumn{2}{|c|}{$\begin{array}{c}\text { 3D- } \\
\text { eigenvector }\end{array}$} & \multicolumn{2}{|c|}{$\begin{array}{c}\text { PPP- } \\
\text { eigenvector }\end{array}$} & \multicolumn{2}{|c|}{$\begin{array}{l}\text { Orthogonal } \\
\text { regression }\end{array}$} & \multicolumn{2}{|c|}{$\begin{array}{l}\text { Nonlinear } \\
\text { regression }\end{array}$} & \multirow{2}{*}{$\begin{array}{c}\mathrm{SH} \\
\text { linear } \\
\text { conv } \\
\end{array}$} & \multirow{2}{*}{$\begin{array}{c}\begin{array}{c}\mathrm{SH} \\
\text { nonlin }\end{array} \\
\text { conv }\end{array}$} \\
\hline$\#$ & name & conv & ideal & conv & ideal & conv & ideal & conv & ideal & conv & ideal & & \\
\hline 1 & $A a 1$ & 1 & 1 & 1 & 2 & 1 & 2 & 1 & 1 & 1 & 2 & 1 & 1 \\
\hline 2 & $A a 2$ & 10 & 18 & 13 & 16 & 10 & 15 & 5 & 7 & 11 & 13 & 13 & 8 \\
\hline 3 & $A a 3$ & 11 & 20 & 6 & 13 & 9 & 14 & 4 & 6 & 12 & 14 & 14 & 13 \\
\hline 4 & $A b 1$ & 7 & 4 & 15 & 10 & 21 & 12 & 8 & 5 & 20 & 18 & 6 & 20 \\
\hline 5 & $A b 2$ & 26 & 15 & 27 & 20 & 27 & 18 & 24 & 23 & 26 & 22 & 19 & 26 \\
\hline 6 & $A b 3$ & 16 & 7 & 17 & 11 & 19 & 11 & 16 & 14 & 32 & 29 & 10 & 32 \\
\hline 7 & $A b 4$ & 17 & 8 & 10 & 5 & 12 & 5 & 22 & 19 & 13 & 8 & 11 & 12 \\
\hline 8 & $A b 5$ & 27 & 21 & 26 & 19 & 29 & 22 & 31 & 30 & 30 & 28 & 21 & 30 \\
\hline 9 & $A c 1$ & 15 & 9 & 14 & 8 & 15 & 9 & 13 & 12 & 10 & 6 & 8 & 10 \\
\hline 10 & $A c 2$ & 22 & 14 & 24 & 17 & 25 & 13 & 27 & 26 & 21 & 17 & 16 & 21 \\
\hline 11 & $A c 3$ & 5 & 3 & 4 & 3 & 6 & 3 & 3 & 3 & 4 & 3 & 4 & 4 \\
\hline 12 & Ac4 & 13 & 6 & 7 & 4 & 11 & 4 & 19 & 15 & 6 & 5 & 7 & 6 \\
\hline 13 & $A c 5$ & 25 & 16 & 18 & 12 & 17 & 10 & 25 & 25 & 18 & 16 & 18 & 19 \\
\hline 14 & $A d 1$ & 3 & 2 & 2 & 1 & 2 & 1 & 6 & 4 & 2 & 1 & 2 & 2 \\
\hline 15 & $A d 2$ & 9 & 12 & 8 & 9 & 8 & 7 & 20 & 21 & 16 & 12 & 9 & 16 \\
\hline 16 & $A d 3$ & 20 & 17 & 16 & 15 & 18 & 16 & 23 & 24 & 15 & 11 & 17 & 15 \\
\hline 17 & $A d 4$ & 12 & 13 & 5 & 7 & 7 & 6 & 21 & 22 & 3 & 4 & 12 & 3 \\
\hline 18 & $A e 1$ & 4 & 5 & 3 & 6 & 3 & 8 & 7 & 8 & 7 & 7 & 3 & 7 \\
\hline 19 & $A e 2$ & 29 & 28 & 22 & 23 & 23 & 23 & 28 & 27 & 24 & 24 & 26 & 24 \\
\hline 20 & $A e 3$ & 30 & 29 & 20 & 22 & 24 & 24 & 29 & 28 & 25 & 25 & 27 & 25 \\
\hline 21 & $A f 1$ & 8 & 10 & 12 & 14 & 16 & 17 & 11 & 10 & 9 & 10 & 15 & 11 \\
\hline 22 & $A f 2$ & 23 & 22 & 28 & 26 & 20 & 19 & 10 & 9 & 28 & 27 & 25 & 28 \\
\hline 23 & $A f 3$ & 21 & 19 & 21 & 21 & 28 & 21 & 12 & 11 & 23 & 21 & 23 & 23 \\
\hline 24 & $B x 1$ & 2 & 11 & 9 & 18 & 5 & 20 & 2 & 2 & 5 & 9 & 5 & 5 \\
\hline 25 & $B \times 2$ & 18 & 25 & 23 & 27 & 14 & 26 & 14 & 16 & 19 & 23 & 22 & 18 \\
\hline 26 & $B \times 3$ & 19 & 26 & 25 & 28 & 26 & 28 & 15 & 18 & 22 & 26 & 24 & 22 \\
\hline 27 & $B \times 4$ & 14 & 24 & 19 & 24 & 13 & 25 & 17 & 20 & 8 & 15 & 20 & 9 \\
\hline 28 & By 1 & 24 & 31 & 29 & 32 & 22 & 29 & 26 & 29 & 27 & 30 & 31 & 27 \\
\hline 29 & By 2 & 6 & 23 & 11 & 25 & 4 & 27 & 9 & 13 & 14 & 19 & 28 & 14 \\
\hline 30 & $B z 1$ & 28 & 27 & 30 & 29 & 31 & 31 & 18 & 17 & 29 & 31 & 29 & 29 \\
\hline 31 & $B z 2$ & 31 & 30 & 31 & 30 & 30 & 30 & 30 & 31 & 17 & 20 & 30 & 17 \\
\hline 32 & $B z 3$ & 33 & 33 & 33 & 33 & 32 & 32 & 32 & 32 & 33 & 33 & 33 & 33 \\
\hline 33 & $B z 4$ & 32 & 32 & 32 & 31 & 33 & 33 & 33 & 33 & 31 & 32 & 32 & 31 \\
\hline
\end{tabular}


The ranks from 1 to 33 are assigned to the alternatives from the best to the worst in each priority vector. By Tables 2 and 3, the conventional mode usually produces more variability in the priority elements, while the ideal mode makes more evenly distributed elements with less rank reversals. The results of the orthogonal regression raise some doubts because they differ noticeably from the other evaluations having a large weight of the first alternative. The preferences in PPP are more evenly allocated to the alternatives, so it is more difficult to distinguish them. Both orthogonal and PPP techniques produce poor approximation results which can also be seen by their small $R^{2}$ in Table 2 .

In spite of some volatility in ranks, the general structure of all the vectors is pretty stable - Table 4 presents a matrix of pair correlations of all the vectors from Table 2, together with the additional rows of the means and totals of the correlations in each column.

Table 4: Correlations between Global Priority Vectors

\begin{tabular}{|c|c|c|c|c|c|c|c|c|c|c|c|c|}
\hline & $\begin{array}{l}\text { Mult } \\
\text { conv }\end{array}$ & $\begin{array}{l}\text { Mult } \\
\text { ideal }\end{array}$ & $\begin{array}{c}3 \mathrm{D} \\
\text { conv }\end{array}$ & $\begin{array}{c}\text { 3D } \\
\text { ideal }\end{array}$ & $\begin{array}{l}\text { PPP } \\
\text { conv }\end{array}$ & $\begin{array}{l}\text { PPP } \\
\text { ideal }\end{array}$ & $\begin{array}{l}\text { Orth } \\
\text { conv }\end{array}$ & $\begin{array}{l}\text { Orth } \\
\text { ideal }\end{array}$ & $\begin{array}{l}\text { NLin } \\
\text { conv }\end{array}$ & $\begin{array}{l}\text { NLin } \\
\text { ideal }\end{array}$ & $\begin{array}{l}\text { SH } \\
\text { lin }\end{array}$ & $\begin{array}{l}\text { SH } \\
\text { nlin }\end{array}$ \\
\hline $\begin{array}{l}\text { Mult } \\
\text { conv }\end{array}$ & 1.00 & 0.82 & 0.90 & 0.66 & 0.86 & 0.57 & 0.90 & 0.89 & 0.88 & 0.70 & 0.95 & 0.88 \\
\hline $\begin{array}{l}\text { Mult } \\
\text { ideal }\end{array}$ & 0.82 & 1.00 & 0.87 & 0.92 & 0.76 & 0.87 & 0.71 & 0.74 & 0.83 & 0.83 & 0.93 & 0.82 \\
\hline $\begin{array}{c}\text { conv } \\
3 \mathrm{D}\end{array}$ & 0.90 & 0.87 & 1.00 & 0.87 & 0.95 & 0.79 & 0.75 & 0.75 & 0.92 & 0.85 & 0.93 & 0.92 \\
\hline $\begin{array}{l}\text { ideal } \\
\text { PPP }\end{array}$ & 0.66 & 0.92 & 0.87 & 1.00 & 0.77 & 0.97 & 0.52 & 0.54 & 0.82 & 0.91 & 0.82 & 0.81 \\
\hline $\begin{array}{l}\text { conv } \\
\text { PPP }\end{array}$ & 0.86 & 0.76 & 0.95 & 0.77 & 1.00 & 0.73 & 0.66 & 0.66 & 0.85 & 0.76 & 0.85 & 0.85 \\
\hline $\begin{array}{l}\text { ideal } \\
\text { Orth }\end{array}$ & 0.57 & 0.87 & 0.79 & 0.97 & 0.73 & 1.00 & 0.41 & 0.44 & 0.71 & 0.84 & 0.75 & 0.71 \\
\hline conv & 0.90 & 0.71 & 0.75 & 0.52 & 0.66 & 0.41 & 1.00 & 0.99 & 0.78 & 0.58 & 0.87 & 0.78 \\
\hline ideal & 0.89 & 0.74 & 0.75 & 0.54 & 0.66 & 0.44 & 0.99 & 1.00 & 0.79 & 0.60 & 0.87 & 0.78 \\
\hline $\begin{array}{l}\text { NLin } \\
\text { conv }\end{array}$ & 0.88 & 0.83 & 0.92 & 0.82 & 0.85 & 0.71 & 0.78 & 0.79 & 1.00 & 0.93 & 0.89 & 1.00 \\
\hline $\begin{array}{c}\text { NLin } \\
\text { ideal } \\
\text { SH }\end{array}$ & 0.70 & 0.83 & 0.85 & 0.91 & 0.76 & 0.84 & 0.58 & 0.60 & 0.93 & 1.00 & 0.79 & 0.92 \\
\hline $\begin{array}{l}\text { Lin } \\
\text { SH }\end{array}$ & 0.95 & 0.93 & 0.93 & 0.82 & 0.85 & 0.75 & 0.87 & 0.87 & 0.89 & 0.79 & 1.00 & 0.89 \\
\hline NLin & 0.88 & 0.82 & 0.92 & 0.81 & 0.85 & 0.71 & 0.78 & 0.78 & 1.00 & 0.92 & 0.89 & 1.00 \\
\hline mean & 0.83 & 0.84 & 0.87 & 0.80 & 0.81 & 0.73 & 0.74 & 0.75 & 0.87 & 0.81 & 0.88 & 0.86 \\
\hline total & 10.00 & 10.10 & 10.50 & 9.61 & 9.70 & 8.78 & 8.94 & 9.05 & 10.39 & 9.72 & 10.54 & 10.36 \\
\hline
\end{tabular}

Table 4 shows that the vectors correspond one to the other very well - the correlations generally are pretty high. The mean level of correlations is about 0.8 and more for most of the vectors. The last row of totals in the columns allows one to easily find which estimations produce more similar vectors. The totals are higher for the Multiplicative in both modes, 3D-eigenvector and Nonlinear estimation in the conventional mode, and Synthetic hierarchy estimation in the linear and nonlinear evaluations. Similar results were 
obtained by the normalized projections (cosines) between the vectors, and by Spearman's rank correlation and Kendall's tau-coefficients constructed by the ranks from Table 3. To finalize priority consideration, the following procedure can be added: calculate the average of all the vectors in Table 2 using weights of the $R^{2}$ shares as a measure of each vector importance - the results are presented in Table 5.

Table 5: Averaged Global Priorities and their Ranks

\begin{tabular}{|c|c|c|c|c|c|}
\hline \multicolumn{3}{|c|}{ Weighted Mean Priorities } & \multicolumn{3}{|c|}{ Priorities Ordered by Rank } \\
\hline item & priority & rank & item & priority & rank \\
\hline$A a 1$ & 15.23 & 1 & $A a 1$ & 15.23 & 1 \\
\hline$A a 2$ & 3.17 & 11 & $A d 1$ & 9.78 & 2 \\
\hline Aa3 & 3.12 & 12 & $A c 3$ & 6.94 & 3 \\
\hline$A b 1$ & 2.80 & 14 & $A d 4$ & 5.57 & 4 \\
\hline$A b 2$ & 1.34 & 22 & $A e 1$ & 5.32 & 5 \\
\hline$A b 3$ & 1.92 & 19 & Ac4 & 4.92 & 6 \\
\hline$A b 4$ & 3.35 & 9 & $B \times 1$ & 4.66 & 7 \\
\hline$A b 5$ & 1.04 & 27 & $A c 1$ & 3.80 & 8 \\
\hline$A c 1$ & 3.80 & 8 & $A b 4$ & 3.35 & 9 \\
\hline$A c 2$ & 1.77 & 20 & $A f 1$ & 3.26 & 10 \\
\hline Ac3 & 6.94 & 3 & $A a 2$ & 3.17 & 11 \\
\hline$A c 4$ & 4.92 & 6 & $A a 3$ & 3.12 & 12 \\
\hline$A c 5$ & 2.04 & 18 & $A d 2$ & 2.99 & 13 \\
\hline$A d 1$ & 9.78 & 2 & $A b 1$ & 2.80 & 14 \\
\hline$A d 2$ & 2.99 & 13 & $A d 3$ & 2.38 & 15 \\
\hline$A d 3$ & 2.38 & 15 & $B \times 4$ & 2.35 & 16 \\
\hline$A d 4$ & 5.57 & 4 & By 2 & 2.16 & 17 \\
\hline$A e 1$ & 5.32 & 5 & $A c 5$ & 2.04 & 18 \\
\hline$A e 2$ & 1.08 & 26 & $A b 3$ & 1.92 & 19 \\
\hline$A e 3$ & 1.02 & 28 & $A c 2$ & 1.77 & 20 \\
\hline$A f 1$ & 3.26 & 10 & $A f 3$ & 1.46 & 21 \\
\hline$A f 2$ & 1.27 & 24 & $A b 2$ & 1.34 & 22 \\
\hline$A f 3$ & 1.46 & 21 & $B \times 2$ & 1.34 & 23 \\
\hline$B x 1$ & 4.66 & 7 & $A f 2$ & 1.27 & 24 \\
\hline$B \times 2$ & 1.34 & 23 & $B \times 3$ & 1.10 & 25 \\
\hline$B \times 3$ & 1.10 & 25 & $A e 2$ & 1.08 & 26 \\
\hline$B \times 4$ & 2.35 & 16 & $A b 5$ & 1.04 & 27 \\
\hline By 1 & 0.68 & 30 & $A e 3$ & 1.02 & 28 \\
\hline By 2 & 2.16 & 17 & $B z 2$ & 0.97 & 29 \\
\hline$B z 1$ & 0.58 & 31 & By 1 & 0.68 & 30 \\
\hline$B z 2$ & 0.97 & 29 & $B z 1$ & 0.58 & 31 \\
\hline$B z 3$ & 0.25 & 33 & $B z 4$ & 0.34 & 32 \\
\hline$B z 4$ & 0.34 & 32 & $B z 3$ & 0.25 & 33 \\
\hline
\end{tabular}

The mean priorities are very similar to most of the estimations in Table 2, but they are more stable and provide an easier way to choose the best items: the most preferred alternatives are $A a 1, A d 1, A c 3$, etc. 


\section{Summary}

The problem of synthesizing the local preferences into global priorities in group decision making by the hierarchy structure is considered. Classic methods are tried together with new developed techniques, including multi-response multiplicative mode, three-dimensional eigenproblem and its simplification in parallel proportional profile estimation, orthogonal regression, non-linear local evaluation, linear and nonlinear synthetic hierarchy optimizing procedures. Marketing research data with 2 criteria, 9 subcriteria, and 33 alternatives was used to produce results by a dozen different approaches. The main estimation techniques yield rather similar global priority vectors with comparable ranks of the items. The most consistent methods are identified for practical managerial decision making in complex hierarchies.

\section{REFERENCES}

Aczel, J., \& Saaty ,T.L. (1983). Procedures for synthesizing ratio judgments, Mathematical Psychology, 27, 93-102.

Barzilai, J., \& Lootsma, F.A. (1997). Power relations and group aggregation in the multiplicative AHP and SMART, Multi-Criteria Decision Analysis, 6, 155 - 165.

Basak, I., \& Saaty, T.L. (1993). Group decision making using the Analytic Hierarchy Process, Mathematical and Computer Modelling, 17, 101-110.

Cattell R.B. (1944). "Parallel Proportional Profiles" and other principles for determining the choice of factors by rotation, Psychometrika, 9, 267-283.

Enea, M., \& Piazza, T. (2004). Project selection by constrained fuzzy AHP, Fuzzy Optimization and Decision Making, 3, 39-62.

Gass, S.I., \& Rapcsak, T. (2004). Singular value decomposition in AHP, European Journal of Operational Research, 154, 573-584.

Golden, B.L., Wasil, E.A., \& Harker, P.T., eds. (1989). The Analytic Hierarchy Process: Applications and Studies. Berlin-Heidelberg: Springer.

Law, H.G., Snyder, C.W., Hattie, J.A., \& McDonald, R.P., eds. (1984). Research methods for multimode data analysis. Praeger: New York.

Lipovetsky, S. (1994). Multimode data analysis for decision making, in: Tzeng G.H., et al., eds. Multiple Criteria Decision Making, 275-282. Springer: New York.

Lipovetsky, S. (1996). The synthetic hierarchy method: An optimizing approach to obtaining priorities in the AHP, European Journal of Operational Research, 93, 550 - 564.

Lipovetsky, S. (2005). Analytic Hierarchy Processing in Chapman-Kolmogorov equations, International Journal of Operations and Quantitative Management, 11, 219-228.

Lipovetsky, S. (2009). Global Priority Estimation in Multiperson Decision Making, Journal of Optimization Theory and Applications, 140, 77-91. 
Lipovetsky, S., \& Conklin, M. (2001). Dual priority-antipriority Thurstone scales as AHP eigenvectors, Engineering Simulation, 18, 631-648.

Lipovetsky, S., \& Conklin, M. (2002). Robust estimation of priorities in the AHP, European Journal of Operational Research, 137, 110-122.

Lipovetsky, S., \& Conklin, M. (2004). Thurstone scaling via binary response regression, Statistical Methodology, 1, 93-104.

Lipovetsky, S., \& Tishler, A. (1994). Linear methods in multimode data analysis for decision making, Computers and Operations Research, 21, 169-183.

Lipovetsky, S., \& Tishler, A. (1999). Interval estimation of priorities in the AHP, European Journal of Operational Research, 114, 153-164.

Lipovetsky, S., Tishler, A., Dvir, D., \& Shenhar, A. (1997). The relative importance of project success dimensions, $R \& D$ Management, 27, 97-106.

MakKay, D.B., Bowen, W.M., \& Zinnes, J.L. (1996). A thurstonian view of the analytic hierarchy process, European Journal of Operational Research 89, 427-444.

Maronna, R. (2005). Principal components and orthogonal regression based on robust scales, Technometrics, 47, 264-273.

Saaty, T.L. (1977). A scaling method for priorities in hierarchical structures, Mathematical Psychology, $15,234-281$.

Saaty, T.L. (1980). The Analytic Hierarchy Process. McGraw-Hill: New York.

Saaty, T.L. (1994). Fundamentals of Decision Making and Priority Theory with the Analytic Hierarchy Process. RWS Publications: Pittsburgh.

Saaty, T.L. (1996). Decision Making with Dependence and Feedback: The Analytic Network Process. RWS Publications: Pittsburgh.

Saaty, T.L. (2000). Decision Making for Leaders. RWS Publications: Pittsburgh.

Saaty, T.L., \& Kearns, K.P. (1985). Analytical Planning. Pergamon Press: New York.

Saaty, T.L., \& Vargas, L.G. (1984). Comparison of eigenvalue, logarithmic least squares and least squares methods in estimating ratios, Mathematical Modelling, 5, 309-324.

Saaty, T.L., \& Vargas, L.G. (1994). Decision Making in Economic, Political, Social and Technological Environment with the Analytic Hierarchy Process. RWS Publications: Pittsburgh.

Tucker, L.R. (1966). Some mathematical notes on three-mode factor analysis. Psychometrika, 31, 279311.

Wasil, E.A., \& Golden, B.L. (2003). Celebrating 25 years of AHP-based decision making, Computers and Operations Research, 30, 1419-1420. 